\title{
Neutrino masses and GUT baryogenesis
}

\section{Juan A. Lopez-Perez*, Nuria Rius}

IFIC Valencia

E-mail: juanlope@ific.uv.es, nuria@ific.uv.es

\begin{abstract}
We reconsider the GUT-baryogenesis mechanism for generating the baryon asymmetry of the Universe. The baryon asymmetry is produced by the out of equilibrium decay of coloured Higgs bosons at the GUT scale, conserving B-L. If neutrinos are Majorana particles, lepton number violating interactions erase the lepton number excess, but part of the baryon asymmetry may be preserved, provided those interactions are not in thermal equilibrium when the sphaleron processes become effective, at $T \sim 10^{12} \mathrm{GeV}$. We analyse whether this mechanism for baryogenesis is feasible in a variety of GUT models of fermion masses proposed in the literature, based on horizontal symmetries.
\end{abstract}

\section{Introduction}

In the GUT-baryogenesis mechanism [1] the baryon asymmetry is generated during the out of equilibrium decay of coloured Higgs bosons, because those decays violate $B$ and $L$. The problem is that in $S U(5)$ grand unification, and in any grand unified model containing $U(1)_{B-L}$ as a subgroup, the dominant Higgs decays conserve $(B-L)$. Below $10^{12} \mathrm{GeV}$ the sphaleron configurations [2] are in thermal equilibrium [3] and violate also $B$ and $L$, but conserve $(B-L)$, relating these quantities as

$$
B=c(B-L)=\frac{c}{c-1} L
$$

where $\mathrm{c}$ is a constant. As a result the baryon asymmetry generated in the Higgs decays is erased, since $(B-L)=0$. However, recently it has been proposed a modified GUT baryogenesis mechanism which may produce the observed baryon asymmetry, provided neutrinos are Majorana particles 沟.

It is now confirmed that the deficits in atmospheric muon neutrinos [0, [] and in solar electron neutrinos [7, 8], as well as the KamLAND observation [9] of electron antineutrino disappearance, are mainly due to neutrino oscillations and therefore neutrinos are massive. One of the simplest ways to generate the small neutrino masses required is the seesaw

\footnotetext{
${ }^{*}$ Speaker.
} 
mechanism [10], in which three heavy singlet right-handed neutrinos, $\nu_{R}$, are added to the Standard Model (SM) particle content. The Lagrangian giving rise to fermion masses is:

$$
\mathcal{L}=\mathcal{L}_{S M}+Y_{\nu} \overline{l_{L}} H \nu_{R}+\frac{1}{2} \overline{\nu_{R}} m_{R} \nu_{R}^{c}+\text { h.c. },
$$

where $\mathcal{L}_{S M}$ stands for the usual SM Yukawa terms, $l_{L}$ are the left-handed lepton doublets (we have suppressed family indices), $H$ is the Higgs doublet, $Y_{\nu}$ are the new Yukawa couplings that generate the neutrino Dirac masses after electroweak symmetry breaking and $m_{R}$ is a $3 \times 3$ Majorana mass matrix.

We expect the overall scale of $m_{R}$ to be much larger than the electroweak scale, because $\nu_{R}$ Majorana masses are $S U(3) x S U(2) x U(1)$ invariant and therefore naturally of the order of the cutoff of the low energy theory. Thus, we can integrate the $\nu_{R}$ away and we get an effective Lagrangian which contains a Majorana mass term for the left-handed neutrinos,

$$
\mathcal{L}_{\text {eff }}=\frac{1}{2} \overline{\nu_{L}} m_{\nu} \nu_{L}^{c}+\text { h.c. },
$$

with

$$
m_{\nu} \simeq m_{D} m_{R}^{-1} m_{D}^{T}
$$

where $m_{D}=Y_{\nu} v$ and $v$ is the VEV of the Higgs doublet. If there is no quasi-degeneracy in the light neutrino masses, we can estimate the heaviest neutrino mass as

$$
m_{h} \sim \sqrt{\Delta m_{a t m}^{2}} \simeq 5 \times 10^{-2} \mathrm{eV},
$$

so for $v \lesssim O(100 \mathrm{GeV})$ and $Y_{\nu} \sim O(1)$ the $\nu_{R}$ mass scale is $\Lambda \lesssim 10^{15} \mathrm{GeV}$, remarkably close to $M_{G U T}$.

As a consequence of this high scale, lepton number violating scattering processes mediated by the heavy right-handed neutrinos

$$
l_{L}+H \rightarrow \nu_{R} \rightarrow \overline{l_{L}}+\bar{H}
$$

are in thermal equilibrium above the temperature $T \sim 10^{14} \mathrm{GeV}$, when the sphaleron interactions are not yet effective. In this new GUT baryogenesis scenario [ [ decay of coloured Higgs bosons produces baryon and lepton asymmetries, conserving $B-$ $L$, but the Majorana interactions erase the lepton number excess before the sphaleron interactions come into thermal equilibrium, at $T_{s p h} \sim 10^{12} \mathrm{GeV}$. At lower temperatures, although the SM $B+L$ violating processes convert baryon number into lepton number, part of the original baryon asymmetry survives, because now $B-L \neq 0$.

In general, for this GUT baryogenesis mechanism to work we should require that $\Gamma_{\Delta L=2}\left(T_{s p h}\right)<H\left(T_{s p h}\right)$, where

$$
\Gamma_{\Delta L=2}(T)=\frac{T^{3}}{4 \pi^{3} v^{4}} \sum_{i=1}^{3} m_{i}^{2}
$$

is the rate of the scattering processes (1.6) and $H(T) \sim 17 T^{2} / M_{\text {Plank }}$ is the Hubble parameter at the temperature $T$. Thus, if the light left-handed neutrinos are degenerate, 
since the rate of the lepton number violating scattering (1.6) is proportional to the sum of the squared neutrino masses, the condition that it is out of equilibrium at $T \lesssim 10^{12} \mathrm{GeV}$ directly translates into an upper bound on the neutrino masses, namely $m_{i} \lesssim 0.2 \mathrm{eV}$ for $v \sim 174 \mathrm{GeV}$.

Even if the lepton number violating scattering is out of equilibrium, for right-handed neutrinos lighter than $\sim 10^{12} \mathrm{GeV}$ we should also require that their lepton number violating decays

$$
\nu_{R} \rightarrow \overline{l_{L}}+\bar{H}
$$

are out of equilibrium at $T \lesssim 10^{12} \mathrm{GeV}$, otherwise these interactions together with the sphalerons would wash out the baryon asymmetry produced in the Higgs decays. For each heavy neutrino, this decay rate is proportional to the mass parameter

$$
\tilde{m}_{i}=\frac{\left(Y_{\nu}^{\dagger} Y_{\nu}\right)_{i i}}{m_{\nu_{R_{i}}}} v^{2}, \quad i=1,2,3
$$

where $m_{\nu_{R_{i}}}$ are the eigenvalues of the Majorana mass matrix $m_{R}$. Notice that while the $\Delta L=2$ scattering rate depends only on the light neutrino masses, the decay rate is model dependent.

Two comments are in order. First, the constraint that the coloured Higgs should decay before the sphaleron processes enter into thermal equilibrium implies that $m_{H_{c}}>$ $10^{12} \mathrm{GeV}$, which is in agreement with the limits from proton decay. Second, in principle this GUT baryogenesis mechanism is also valid for supersymmetric models, however the high temperature at which the baryon asymmetry is produced $\left(T>10^{12} \mathrm{GeV}\right)$ may be a problem in inflationary cosmology, since it is well above the upper bound on the reheat temperature of the Universe from gravitino production.

We have addressed the question of whether this scenario for baryogenesis can be realised in several Grand Unified models of fermion masses proposed in the literature. More precisely, we focus on models with horizontal symmetries in which neutrino masses are generated via the seesaw mechanism. Our estimates are also applicable to the supersymmetric versions of these models, with the caveat of the gravitino problem. For a more complete analysis and details, see [11].

\section{Models of Fermion Masses}

Atmospheric and solar neutrino oscillations can be fitted with two small neutrino mass differences, $\Delta m_{\text {atm }}^{2}=2.5 \times 10^{-3} \mathrm{eV}^{2}$ and $\Delta m_{\text {sol }}^{2}=6.9 \times 10^{-5} \mathrm{eV}^{2}$. It is also known from experiment that the atmospheric mixing angle is nearly maximal, $c_{23} \sim s_{23} \sim 1 / \sqrt{2}$, $s_{13}<0.2$ according to CHOOZ [12] and the solar angle is large.

These observations are consistent with three patterns of neutrino masses: hierarchical $\left(\Delta m_{\text {atm }}^{2} \sim m_{3}^{2}, \Delta m_{\text {sol }}^{2} \sim m_{2}^{2}\right)$, inverted hierarchical or quasi-Dirac $\left(\Delta m_{\text {atm }}^{2} \sim m_{3}^{2} \sim m_{2}^{2}\right.$, $\left.\Delta m_{\text {sol }}^{2} \sim m_{3}^{2}-m_{2}^{2}\right)$ and degenerate $\left(m_{3}^{2} \sim m_{2}^{2} \sim m_{1}^{2} \gg \Delta m_{\text {atm }}^{2}\right)$. The absolute scale is unknown, though, since neutrino oscillations are only sensitive to mass differences. In this respect, the search for neutrinoless double beta decay is crucial because it would provide 
information about the absolute spectrum and, moreover, discriminate between Majorana and Dirac neutrinos.

We assume three light Majorana neutrinos, whose masses arise from the minimal seesaw mechanism. As we have seen, in this context the scale $\Lambda$ for $L$ non-conservation is suggestively close to $M_{G U T}$, so we consider Grand Unified models for all fermion masses. Horizontal (or family) symmetries have been proposed with the aim of explaining the hierarchical pattern of quark and charged lepton masses [13]. For simplicity, we have analysed models with abelian $U(1)_{H}$ family symmetry.

The horizontal symmetry allows only third family (or just top quark) Yukawa couplings, however it is spontaneously broken by the vev's $v_{f}$ of some "flavon" fields $\Phi$, which are singlets under the grand unified gauge group but charged under the flavour symmetry. Then smaller couplings are generated effectively from higher dimension non-renormalisable operators but are suppressed by powers of the parameter $v_{f} / \Lambda_{c}$, where $\Lambda_{c}$ is the cut-off of the theory. We expect $v_{f} \gtrsim M_{G U T}$ and $\Lambda_{c} \lesssim M_{\text {Plank }}$, so the ratio $v_{f} / \Lambda_{c}$ can be identified with the Wolfenstein parameter, $\lambda \sim 0.22$. The number of powers of the expansion parameter is controlled by the flavor charge of the particular operator.

Notice that in all the models considered (and in most of this kind existent in the literature) the light neutrino masses are hierarchical, since it is difficult to obtain a degenerate spectrum in the context of GUT models of fermion masses. Then, as we explained before, $\Delta L=2$ scattering processes are always out of equilibrium at $T_{s p h} \sim 10^{12} \mathrm{GeV}$, when the $B-L$ conserving interactions become active. So what we require for the modified GUT baryogenesis to work is that either the right-handed neutrinos are heavier than $10^{12} \mathrm{GeV}$, and thus they are not present in the thermal bath when the sphalerons enter into thermal equilibrium or, if they are lighter, their $\Delta L=1$ decays are out equilibrium at $T \leq T_{s p h}$, i.e., $\Gamma_{\Delta L=1}\left(T=M_{i}\right)<H\left(T=M_{i}\right)$ for $M_{i} \leq 10^{12} \mathrm{GeV}^{1}$.

\section{1 $S U(5) \times U(1)_{H}$ models}

In Grand Unified $S U(5)$ models the SM quarks and leptons are grouped in the multiplets $\mathbf{1 0}=\left(q_{L}, u_{R}^{c}, e_{R}^{c}\right)$ and $\mathbf{5}^{*}=\left(d_{R}^{c}, l_{L}\right)$ and the right-handed neutrinos are added in the singlet representation $\mathbf{1}=\nu_{R}^{c}$. The Higgs multiplets transform as a $\mathbf{5}$, and contain the coloured triplets whose decays generate the baryon asymmetry in the proposed mechanism. We assume that there are two Higgs multiplets because it has been shown that with just one the baryon asymmetry generated is too small [14].

\subsubsection{Q(fermions) $\geq 0$}

First we consider a model in which the $U(1)_{H}$ symmetry is broken by one singlet field $\Phi$ with charge $Q=-1$, and all fermions with non-negative charges [15]. The expansion parameter in this model is chosen to be $\epsilon=\langle\Phi\rangle / \Lambda_{c} \simeq 0.06$ i.e., $\epsilon \simeq \lambda^{2}$. From the observed hierarchy of quark and charged lepton masses one can deduce that the allowed $U(1)_{H}$

\footnotetext{
${ }^{1}$ Note that this condition ensures that $\Gamma_{\Delta L=1}(T)<H(T)$ at any $T>M_{i}$, so it is enough to consider $T=M_{i}$.
} 
charges are

\begin{tabular}{|ccc|ccc|ccc|}
\hline $\mathbf{1 0}_{\mathbf{3}}$ & $\mathbf{1 0}_{\mathbf{2}}$ & $\mathbf{1 0}_{\mathbf{1}}$ & $\mathbf{5}_{\mathbf{3}}^{*}$ & $\mathbf{5}_{\mathbf{2}}^{*}$ & $\mathbf{5}_{\mathbf{1}}^{*}$ & $\mathbf{1}_{\mathbf{3}}$ & $\mathbf{1}_{\mathbf{2}}$ & $\mathbf{1}_{\mathbf{1}}$ \\
\hline \hline 0 & 1 & 2 & $a$ & $a$ & $a+1$ & $b$ & $c$ & $d$ \\
\hline
\end{tabular}

where the value of the $\mathrm{b}$ quark mass allows $a=0,1$ [16] and $0 \leq b \leq c \leq d$.

With those charges one can readily calculate the Dirac neutrino mass matrix and the Majorana mass matrix of the right-handed neutrinos, up to order one coefficients [15]. Then, using the seesaw formula (1.4) one obtains the effective Majorana mass matrix for the light neutrinos:

$$
m_{\nu}=\epsilon^{2 a}\left(\begin{array}{ccc}
\epsilon^{2} & \epsilon & \epsilon \\
\epsilon & 1 & 1 \\
\epsilon & 1 & 1
\end{array}\right) \frac{v^{2}}{\Lambda}
$$

Notice that in this kind of models with one single flavon field and all fermions with non-negative charges the dependence on the $U(1)_{H}$ charges of the right-handed neutrinos drops out, and the light neutrino Majorana mass matrix depends only on the charges of the $\mathbf{5}$ fermions. Thus it has always the hierarchy structure of (2.2), that has been proposed (among others that we will discussed later) to generate naturally the large atmospheric mixing angle. The hierarchy between $m_{2}$ and $m_{3}$ is then considered accidental, since the sub-determinant 23 of $m_{\nu}$ is in general $O(1)$, but this is not unlikely for the LMA solution of the solar neutrino problem: $m_{2} / m_{3} \sim 0.1$ is not particularly small and could easily arise from a combination of order one terms.

We have analysed different values of the $U(1)_{H}$ charges $a, b, c, d$. Clearly, the modified GUT baryogensis scenario requires heavy right-handed neutrinos so the most favourable case is $a=b=c=d=0$, which corresponds to almost degenerate right-handed neutrinos with masses of order $\Lambda \sim 10^{14} \mathrm{GeV}$. Therefore for these $U(1)_{H}$ charges the mechanism is feasible.

The cases $a=1, b=c=d=0$ and $a=b=c=0, d=1$ are somehow in the limit because the lightest right-handed neutrino masses are $M_{i} \sim 10^{12} \mathrm{GeV}$ and we have found that $\Gamma_{\Delta L=1}\left(T=M_{i}\right) \gtrsim H\left(T=M_{i}\right)$. Thus a more detailed analysis would be needed to elucidate these cases.

For all other values of the $U(1)_{H}$ charges, the lightest right-handed neutrino mass is well bellow $10^{12} \mathrm{GeV}$ and its $\Delta L=1$ decays are in equilibrium at $T \lesssim T_{\text {sph }}$ so GUT baryogenesis is not possible.

\subsubsection{Q(fermions) of both signs}

One can also consider that the $U(1)_{H}$ family symmetry is broken by a pair of $S U(5)$ singlets $\Phi$ and $\bar{\Phi}$ with charges $Q=1$ and $Q=-1$, respectively [17]. Taking $\lambda=\langle\Phi\rangle / \Lambda_{c} \sim \lambda^{\prime}=$ $\langle\bar{\Phi}\rangle / \Lambda_{c} \sim 0.22$, the fermion masses can be described by the following set of charges:

\begin{tabular}{|ccc|ccc|ccc|}
\hline $\mathbf{1 0}_{\mathbf{3}}$ & $\mathbf{1 0}_{\mathbf{2}}$ & $\mathbf{1 0}_{\mathbf{1}}$ & $\mathbf{5}_{\mathbf{3}}^{*}$ & $\mathbf{5}_{\mathbf{2}}^{*}$ & $\mathbf{5}_{\mathbf{1}}^{*}$ & $\mathbf{1}_{\mathbf{3}}$ & $\mathbf{1}_{\mathbf{2}}$ & $\mathbf{1}_{\mathbf{1}}$ \\
\hline \hline 0 & 2 & 3 & 0 & 0 & $b$ & 0 & $-a$ & $a$ \\
\hline
\end{tabular}


where $b \geq 2 a>0$. If $b=2$ or 3 , the up, down and charged lepton sectors are not essentially different from the previous case. In the neutrino sector, the Dirac and Majorana mass matrices are given by

$$
m_{D}=\left(\begin{array}{ccc}
\lambda^{a+b} & \lambda^{b-a} & \lambda^{b} \\
\lambda^{a} & \lambda^{\prime a} & 1 \\
\lambda^{a} & \lambda^{\prime a} & 1
\end{array}\right) \quad v \quad m_{R}=\left(\begin{array}{ccc}
\lambda^{2 a} & 1 & \lambda^{a} \\
1 & \lambda^{\prime 2 a} & \lambda^{\prime a} \\
\lambda^{a} & \lambda^{\prime a} & 1
\end{array}\right) \Lambda
$$

The $O(1)$ off-diagonal entry of $m_{D}$ is typical of the so-called lopsided models, which have been proposed to obtain simultaneously the large atmospheric neutrino mixing and the observed mass splitting between the solar and atmospheric frequencies. After diagonalising the charged lepton sector and integrating out the heavy right-handed neutrinos, one obtains the effective Majorana mass matrix for the light neutrinos:

$$
m_{\nu}=\left(\begin{array}{ccc}
\lambda^{2 b} & \lambda^{b} & \lambda^{b} \\
\lambda^{b} & 1+\lambda^{a} \lambda^{\prime a} & 1+\lambda^{a} \lambda^{\prime a} \\
\lambda^{b} & 1+\lambda^{a} \lambda^{\prime a} & 1+\lambda^{a} \lambda^{\prime a}
\end{array}\right) \frac{v^{2}}{\Lambda}
$$

The important property of $m_{\nu}$ is that, as a result of the seesaw mechanism and the $U(1)_{H}$ charge assignments, the determinant of the 23 block is automatically of $O\left(\lambda^{a} \lambda^{\prime a}\right)$, while the $O(1)$ elements lead to large atmospheric mixing angle. This property is entirely due to the specific lopsided form of the Dirac neutrino mass matrix $m_{D}$, for any generic Majorana matrix $m_{R}$, with the only condition that the 33 entry is non vanishing, and that no new $O(1)$ terms are generated in $m_{\nu}$ by a compensation between small terms in $m_{D}$ and large terms in $m_{R}^{-1}$.

For $\lambda \sim \lambda^{\prime}$, it is easy to verify that the eigenvalues of $m_{\nu}$ satisfy the relation $m_{1}: m_{2}$ : $m_{3}=\lambda^{2(b-a)}: \lambda^{2 a}: 1$ and that the solar mixing angle is $\theta_{12} \sim \lambda^{b-2 a}$. Thus, it is possible to reproduce the MSW large mixing angle solution for $b=2$ and $a=1$.

Again, in this type of models the three right-handed neutrinos are heavy, with masses of order $\Lambda \sim 10^{14} \mathrm{GeV}$, and the GUT baryogenesis seems viable.

\section{2 $S O(10)$ models}

It is well known that the three SM families of quarks and leptons together with the extra right-handed neutrinos fit neatly into three copies of the $S O(10)$ spinor representation 16. This feature has made $S O(10)$ one of the most attractive unification groups. Regarding $S O(10)$ Grand Unified models we first consider a detailed top-down model, that fits all fermion masses and mixings, and then we follow a bottom-up approach, which aims to reconstruct the right-handed neutrino Majorana matrix $m_{R}$ from the low energy effective mass matrix $m_{\nu}$, in the framework of a $S O(10)$ inspired pattern for the Dirac neutrino mass matrix, $m_{D}$. In both cases, neutrino masses are generated via the minimal seesaw, without additional Majorana mass terms due to small vevs of $S U(2)$ triplets, which are possible in $S O(10)$ models [18. 


\subsubsection{Albright-Barr model}

This model, developed in [19], is based on a $S O(10)$ gauge group supplemented by a horizontal symmetry $U(1)_{H} \times Z_{2} \times Z_{2}$. It is an attempt to construct a realistic $S O(10)$ model with the minimal Higgs content. The Dirac mass matrices for the neutrinos and charged leptons are

$$
m_{D}=Y_{\nu} M_{U}=\left(\begin{array}{ccc}
\eta & 0 & 0 \\
0 & 0 & -\epsilon \\
0 & \epsilon & 1
\end{array}\right) \quad M_{U} \quad m_{l}=Y_{l} M_{D}=\left(\begin{array}{ccc}
\eta & \delta & \delta^{\prime} e^{i \phi} \\
\delta & 0 & -\epsilon \\
\delta^{\prime} e^{i \phi} & \sigma+\epsilon & 1
\end{array}\right) M_{D}
$$

The zeroes arise from restrictions because of the $U(1) \times Z_{2} \times Z_{2}$ flavour symmetry, which forbids any Froggatt-Nielsen diagram. The antisymmetric $\epsilon$ terms arise from diagrams involving the adjoint $\left\langle\mathbf{4 5}_{\mathbf{H}}\right\rangle$ Higgs vev pointing in the $B-L$ direction. The Dirac mass matrices for the quarks can be found in [19].

The model fits all nine quark and charged lepton masses plus the three CKM angles and $\mathrm{CP}$ phase with the eight input parameters

$$
\begin{array}{cc}
M_{U} \simeq 113 \mathrm{GeV} & M_{D} \simeq 1 \mathrm{GeV} \\
\sigma=1.78 & \epsilon=0.145 \\
\delta=0.0086 & \delta^{\prime}=0.0079 \\
\phi=54^{\circ} & \eta=8 \times 10^{-6}
\end{array}
$$

As a consequence of the lopsided nature of the large $\sigma$ term in the matrix $m_{l}$, the hermitian matrix $m_{l}^{\dagger} m_{l}$ is diagonalised by a large left-handed rotation, which accounts for the near maximal atmospheric neutrino mixing for any reasonable neutrino Majorana mass matrix $m_{R}$.

The type of solar neutrino mixing is determined by the texture of $m_{R}$, which is rather independent of the Dirac mass matrices (2.6) because it arises from completely different operators 19. In particular, the LMA solution of the solar neutrino problem requires a nearly hierarchical texture, namely

$$
m_{R}=\left(\begin{array}{ccc}
c^{2} \eta^{2} & -b \epsilon \eta & a \eta \\
-b \epsilon \eta & d^{2} \epsilon^{2} & -d \epsilon \\
a \eta & -d \epsilon & 1
\end{array}\right) \Lambda
$$

where $\epsilon$ and $\eta$ are specified in eq. (2.7) and $a, b, c, d$ are order 1 parameters restricted by neutrino data. The fact that the same $d$ appears in the 22,23 and 32 elements of $m_{R}$ is due to the factorised structure of the diagrams leading to the right-handed neutrino masses, and therefore is not fine-tuning. However this coefficient is strongly constrained by the CHOOZ bound, $s_{13} \lesssim 0.2$, which requires $0.85 \leq d \leq 1.15$ [19].

Using the seesaw formula with $d=1$ one obtains the effective left-handed neutrino Majorana mass matrix

$$
m_{\nu}=\left(\begin{array}{ccc}
0 & \epsilon /(a-b) & 0 \\
\epsilon /(a-b) & -\epsilon^{2}\left(c^{2}-b^{2}\right) /(a-b)^{2} & -b \epsilon /(a-b) \\
0 & -b \epsilon /(a-b) & 1
\end{array}\right) M_{U}^{2} / \Lambda
$$


which leads to a normal hierarchical spectrum for the light neutrinos.

One of the simplest cases allowed is $a=1, b=c=2$ and $\Lambda=10^{14} \mathrm{GeV}$. Then, the two lightest right-handed neutrinos have masses of order $m_{\nu_{R_{1}}} \sim m_{\nu_{R_{2}}} \sim 10^{8} \mathrm{GeV}$ and we have found that their $\Delta L \neq 0$ decays are in equilibrium below $10^{11} \mathrm{GeV}$, so together with the sphaleron processes they will wash out the baryon asymmetry generated during the Higgs decays. Given the strong hierarchy of $m_{R}$, we expect that this result is generic for any value of the free parameters of the model.

\subsubsection{Branco et al. approach}

The bottom-up approach used in 20] predicts the $\nu_{R}$ masses from $\nu_{L}$ parameters. The $m_{\nu}$ matrix can be written as $m_{\nu}=U_{M N S} d_{\nu} U_{M N S}^{T}$, where we know the mass squared differences and the mixing angles. In the basis where the charged lepton mass matrix $m_{l}$ is real and diagonal, the Dirac neutrino mass matrix can be written as $m_{D}=V_{L}^{\dagger} d_{D} U_{R}$, with $d_{D}$ diagonal and $V_{L}, U_{R}$ unitary matrices. Then, using the seesaw formula

$$
m_{\nu}=m_{D} m_{R}^{-1} m_{D}^{T}=-V_{L}^{\dagger} d_{D} U_{R} m_{R}^{-1} U_{R}^{T} d_{D} V_{L}^{*}
$$

The $V_{L}$ matrix is taken to be the identity in the analytic approach of [20]; this is a reasonable approximation in a minimal $S O(10)$ scenario, where one expects $V_{L}$ to be of the order of the CKM matrix, although it may not be the case in more realistic models. Defining $M \equiv U_{R} m_{R}^{-1} U_{R}^{T}$ so

$$
m_{\nu} \simeq-d_{D} M d_{D}
$$

one gets

$$
M \simeq-d_{D}^{-1} m_{\nu} d_{D}^{-1}
$$

Note that $M$ is $m_{R}^{-1}$ in the basis where the Dirac mass matrix $m_{D}$ is diagonal. Now, using the $S O(10)$ motivated relation $m_{D} \sim m_{u}$ and taking into account the up-quark mass hierarchy at the GUT scale, one has

$$
d_{D} \sim\left(\begin{array}{ccc}
m_{u} & 0 & 0 \\
0 & m_{c} & 0 \\
0 & 0 & m_{t}
\end{array}\right) \simeq\left(\begin{array}{ccc}
\epsilon^{2} & 0 & 0 \\
0 & \epsilon & 0 \\
0 & 0 & 1
\end{array}\right) m_{t}
$$

where $\epsilon \sim \lambda^{4} \simeq 3 \times 10^{-3}$.

The matrix $M$ can be analytically diagonalised at leading order in $\epsilon$ (see ref. 20 for details), and the right-handed neutrino masses are just the inverse of these eigenvalues. As in the previous $S O(10)$ model, the $\nu_{R}$ have a strong hierarchy: $m_{\nu_{R_{1}}}: m_{\nu_{R_{2}}}: m_{\nu_{R_{3}}} \sim$ $\epsilon^{4}: \epsilon^{2}: 1$, so we expect at least one of the right-handed neutrinos to be lighter than $T_{s p h} \sim 10^{12} \mathrm{GeV}$ and, as a consequence, it is unlikely that the GUT baryogenesis works within this framework.

For instance, assuming a hierarchical spectrum of the light left-handed neutrinos, $m_{1} \ll$ $m_{2} \ll m_{3}$, we found

$$
m_{\nu_{R_{1}}} \sim \frac{m_{t}^{2} \epsilon^{4}}{m_{2} \sin ^{2} \theta_{12}} \sim 10^{5} \mathrm{GeV} \quad m_{\nu_{R_{2}}} \sim \frac{2 m_{t}^{2} \epsilon^{2}}{m_{3}} \sim 10^{9} \mathrm{GeV} \quad m_{\nu_{R_{3}}} \sim \frac{m_{t}^{2} \sin ^{2} \theta_{12}}{2 m_{1}}
$$


where we have neglected $s_{13}$ and we have taken $\theta_{12} \sim \pi / 4, m_{3}=\sqrt{\Delta m_{\text {atm }}^{2}} \simeq 5 \times 10^{-2} \mathrm{eV}$ and $m_{2}=\sqrt{\Delta m_{\text {sol }}^{2}} \simeq 8 \times 10^{-3} \mathrm{eV}$.

We have calculated that the $\Delta L=1$ decays of the lightest right-handed neutrinos $\nu_{R_{1,2}}$ are in equilibrium around $10^{6} \mathrm{GeV}$ and $10^{9} \mathrm{GeV}$ respectively, so those decays continue destroying lepton number at $T \lesssim 10^{12} \mathrm{GeV}$ when the sphaleron configurations are also in equilibrium. Then, the baryon asymmetry generated in the coloured Higgs decays is completely washed out. Similar results are obtained if we assume an inverted-hierarchical spectrum of the left-handed neutrinos.

\subsection{Pati-Salam}

In this section we analyse a supersymmetric string-inspired model of all fermion masses and mixing angles based on the Pati-Salam $S U(4) \times S U(2)_{L} \times S U(2)_{R}$ gauge group [21] supplemented by a $U(1)_{H}$ flavour symmetry [22]. It provides an example of a realistic grand unified model in which both the large atmospheric mixing angle and the hierarchical neutrino masses arise naturally, due to the so-called single right-handed neutrino dominance (SRHND) mechanism, that we will discuss in the next section. Here we are just concerned about the neutrino sector, see ref.[22 for details of the full model.

An attractive feature of grand unified models based on the Pati-Salam group is that the presence of the gauged $S U(2)_{R}$ subgroup predicts the existence of the right-handed neutrinos, much as in $S O(10)$. The SM fermions, together with the right-handed neutrinos, are accommodated in the $F=(4,2,1)$ and the $F^{c}=(\overline{4}, 1, \overline{2})$ representations,

$$
F_{A}=\left(\begin{array}{llll}
u & u & u & \nu \\
d & d & d & e
\end{array}\right)_{A} \quad F_{B}^{c}=\left(\begin{array}{llll}
d^{c} & d^{c} & d^{c} & e^{c} \\
u^{c} & u^{c} & u^{c} & \nu^{c}
\end{array}\right)_{B}
$$

and the SM Higgs boson is contained in $h=(1, \overline{2}, 2)$. In order to obtain a realistic SM fermion spectrum, the mass matrices are generated by non-renormalisable operators of the form

$$
F_{A} F_{B}^{c} h\left(\frac{H \bar{H}}{\Lambda_{c}^{2}}\right)^{n}\left(\frac{\theta}{\Lambda_{c}}\right)^{p_{A B}} \quad \text { and } \quad F_{A} F_{B}^{c} h\left(\frac{H \bar{H}}{\Lambda_{c}^{2}}\right)^{n}\left(\frac{\bar{\theta}}{\Lambda_{c}}\right)^{p_{A B}}
$$

where $H=(4,1,2)$ and $\bar{H}=(\overline{4}, 1, \overline{2})$ are the heavy Higgs fields that break the $S U(4) \times$ $S U(2)_{L} \times S U(2)_{R}$ symmetry to the SM group at $M_{G U T}, \theta, \bar{\theta}$ are the Pati-Salam singlets which carry $U(1)_{H}$ charges \pm 1 and break the flavour symmetry, and $\Lambda_{c}>M_{G U T}$ denotes the mass of extra matter that has been integrated out. When the $H$ and $\theta$ fields develop their vev's, those operators reduce to effective Yukawa couplings with powers of the small coefficients

$$
\delta=\frac{\langle H\rangle\langle\bar{H}\rangle}{\Lambda_{c}^{2}} \simeq 0.22 \quad \epsilon=\frac{\langle\theta\rangle}{\Lambda_{c}} \simeq 0.22
$$

Then one can identify $\delta$ with mass splitting within generations and $\epsilon$ with splitting between generations. On the other hand, the right-handed neutrinos $\nu^{c}$ acquire a large mass, $\left(m_{R}\right)_{i j} \sim \delta^{m} \epsilon^{q_{i j}}\langle H H\rangle / \Lambda_{c}$. 
This model predicts the following mass matrices

$$
\begin{aligned}
& m_{D} \sim\left(\begin{array}{lll}
\delta^{3} \epsilon^{5} & \delta \epsilon^{3} & \delta \epsilon \\
\delta^{3} \epsilon^{4} & \delta^{2} \epsilon^{2} & \delta \\
\delta^{3} \epsilon^{4} & \delta^{2} \epsilon^{2} & 1
\end{array}\right) v \sim\left(\begin{array}{ccc}
\lambda^{8} & \lambda^{4} & \lambda^{2} \\
\lambda^{7} & \lambda^{4} & \lambda \\
\lambda^{7} & \lambda^{4} & 1
\end{array}\right) v \\
& m_{R} \sim\left(\begin{array}{ccc}
\delta \epsilon^{8} & \delta \epsilon^{6} & \delta \epsilon^{4} \\
\delta \epsilon^{6} & \delta \epsilon^{4} & \delta \epsilon^{2} \\
\delta \epsilon^{4} & \delta \epsilon^{2} & 1
\end{array}\right) \Lambda \sim\left(\begin{array}{ccc}
\lambda^{9} & \lambda^{7} & \lambda^{5} \\
\lambda^{7} & \lambda^{5} & \lambda^{3} \\
\lambda^{5} & \lambda^{3} & 1
\end{array}\right) \Lambda
\end{aligned}
$$

Then, using the seesaw mechanism (1.4) one can calculate the effective Majorana mass matrix $m_{\nu}$,

$$
m_{\nu} \sim\left(\begin{array}{ccc}
\lambda^{2} & \lambda^{3 / 2} & \lambda^{3 / 2} \\
\lambda^{3 / 2} & 1 & 1 \\
\lambda^{3 / 2} & 1 & 1
\end{array}\right) \frac{v^{2}}{\Lambda}
$$

which is dominated by the third right-handed neutrino.

Requiring that the heaviest mass eigenvalue is $m_{3}=\sqrt{\Delta m_{\text {atm }}^{2}} \simeq 5 \times 10^{-2} \mathrm{eV}$, the mass scale of the right-handed neutrinos results to be $\Lambda \lesssim 6 \times 10^{14} \mathrm{GeV}$ so the two lightest $\nu_{R}$ 's have masses $m_{\nu_{R_{1}}} \lesssim 7 \times 10^{8} \mathrm{GeV}, m_{\nu_{R_{2}}} \lesssim 3 \times 10^{11} \mathrm{GeV}$. Moreover, we have calculated that their $\Delta L=1$ decays are in equilibrium around $10^{9} \mathrm{GeV}$ for $\nu_{R_{1}}$ and $7 \times 10^{11} \mathrm{GeV}$ for $\nu_{R_{2}}$, so these decays continue destroying lepton number at $T \lesssim 10^{12} \mathrm{GeV}$, with the sphaleron configurations in equilibrium, and the baryon asymmetry that could be generated in the heavy Higgs decays disappears. Therefore the GUT baryogenesis mechanism does not work within this model.

\subsection{SRHND}

The single right-handed neutrino dominance (SRHND) [23 is an alternative mechanism proposed in the literature to generate simultaneously a large atmospheric mixing angle, $\theta_{23}$, and hierarchical left-handed neutrino masses. The idea is that the dominant contributions to the 23 block of the light effective Majorana mass matrix $m_{\nu}$ come from only one of the right-handed neutrinos, leading to an approximately vanishing 23 sub-determinant. Next to leading contributions from the other right-handed neutrinos are required to generate the mass splitting and large 12 mixing angle corresponding to the LMA solution of the solar neutrino puzzle.

This mechanism can be realised in many different models, in particular it has been thoroughly studied in the framework of $U(1)_{H}$ flavor symmetry models. These can be embedded in grand unified theories by imposing the corresponding constraints on the quark and lepton $U(1)_{H}$ charges, although usually the minimal models are difficult to reconcile with the data. Therefore in order to analyse if GUT baryogenesis is possible when SRHND takes place, we shall just study different charge assignments to the leptonic sector which 
reproduce the measured neutrino mass splittings and mixing angles, without specifying the quark charges corresponding to a particular grand unified group.

If we take $\nu_{R_{3}}$, of mass $Y$, to be the dominant right-handed neutrino, there are three possible textures of the heavy Majorana mass matrix $m_{R}$ that maintain its isolation from the other right-handed neutrinos, namely the diagonal, democratic and off-diagonal textures [23]

$$
m_{R}^{\text {diag }}=\left(\begin{array}{ccc}
X^{\prime} & 0 & 0 \\
0 & X & 0 \\
0 & 0 & Y
\end{array}\right) \quad m_{R}^{\text {dem }}=\left(\begin{array}{ccc}
X & X & 0 \\
X & X & 0 \\
0 & 0 & Y
\end{array}\right) \quad m_{R}^{\text {off-diag }}=\left(\begin{array}{ccc}
0 & X & 0 \\
X & 0 & 0 \\
0 & 0 & Y
\end{array}\right)
$$

In the democratic and off-diagonal textures, the Majorana masses of the upper block, $X$, are of the same order but not exactly equal.

Denoting the Dirac neutrino mass matrix $m_{D}$ as

$$
m_{D}=\left(\begin{array}{lll}
a^{\prime} & a & d \\
b^{\prime} & b & e \\
c^{\prime} & c & f
\end{array}\right) v
$$

and using the seesaw formula, one obtains the effective light neutrino Majorana mass matrix, $m_{\nu}$. The conditions for SRHND are that the $1 / Y$ terms in the 23 sub-matrix dominate over the $1 / X, 1 / X^{\prime}$ terms in the full matrix,

$$
\frac{e^{2}}{Y} \sim \frac{e f}{Y} \sim \frac{f^{2}}{Y} \gg \mathcal{O}\left(\frac{1}{X}\right), \mathcal{O}\left(\frac{1}{X^{\prime}}\right)
$$

At order $\mathcal{O}\left(\frac{1}{Y}\right)$ the matrix $m_{\nu}$ is the same for the three textures, leading to the heaviest neutrino mass

$$
m_{\nu_{3}}=\frac{d^{2}+e^{2}+f^{2}}{Y} v^{2}
$$

and the mixing angles 23]

$$
\tan \theta_{23}=\frac{e}{f} \quad \tan \theta_{13}=\frac{d}{\sqrt{e^{2}+f^{2}}}
$$

Taking into account that $\tan \theta_{23} \approx 1$ from Super-Kamiokande and $\tan \theta_{13} \lesssim 0.2$ from CHOOZ [12], it is necessary that $d \ll e \approx f[23]$.

The masses of the two remaining neutrinos as well as the solar mixing angle $\theta_{12}$ appear at subleading order, and their analytic expressions depend on the particular texture of $m_{R}$. In the diagonal case, if $|x y| / X \gg\left|x^{\prime} y^{\prime}\right| / X^{\prime}\left(x, y=a, b, c\right.$ and $\left.x^{\prime}, y^{\prime}=a^{\prime}, b^{\prime}, c^{\prime}\right)$, we have $m_{\nu_{1}} \ll m_{\nu_{2}}$, while the diagonal texture when both $|x y| / X$ and $\left|x^{\prime} y^{\prime}\right| / X^{\prime}$ terms are important, as well as the other two textures, lead to $m_{\nu_{1}} \sim m_{\nu_{2}}$.

In 23] it has been shown that the conditions for SRHND and LMA solution of the solar neutrino deficit can be fulfilled in the framework of models with $U(1)_{H}$ family symmetries, with appropriate charge assignments, and a systematic search of the simplest examples has been performed. They assume that the $U(1)_{H}$ is broken by the equal vevs of two singlets, 
$\Phi, \bar{\Phi}$, which have charges \pm 1 , so both signs for the charges of the leptons are allowed. Then, omitting order one coefficients as usual, the Dirac neutrino mass matrix and the heavy Majorana matrix are

$$
\begin{gathered}
m_{D}=\left(\begin{array}{ccc}
\lambda^{\left|n_{1}+l_{1}\right|} & \lambda^{\left|n_{2}+l_{1}\right|} & \lambda^{\left|n_{3}+l_{1}\right|} \\
\lambda^{\left|n_{1}+l_{2}\right|} & \lambda^{\left|n_{2}+l_{2}\right|} & \lambda^{\left|n_{3}+l_{2}\right|} \\
\lambda^{\left|n_{1}+l_{3}\right|} & \lambda^{\left|n_{2}+l_{3}\right|} & \lambda^{\left|n_{3}+l_{3}\right|}
\end{array}\right) v \\
m_{R}=\left(\begin{array}{ccc}
\lambda^{\left|2 n_{1}+\sigma\right|} & \lambda^{\left|n_{2}+n_{1}+\sigma\right|} & \lambda^{\left|n_{3}+n_{1}+\sigma\right|} \\
\lambda^{\left|n_{1}+n_{2}+\sigma\right|} & \lambda^{\left|2 n_{2}+\sigma\right|} & \lambda^{\left|n_{3}+n_{2}+\sigma\right|} \\
\lambda^{\left|n_{1}+n_{3}+\sigma\right|} & \lambda^{\left|n_{2}+n_{3}+\sigma\right|} & \lambda^{\left|2 n_{3}+\sigma\right|}
\end{array}\right) \Lambda
\end{gathered}
$$

where the charges $l_{i}, n_{i}, \sigma$ correspond to the $l_{L}, \nu_{R}$ and $S$ fields, being $S$ the one whose vev generates the heavy Majorana neutrino masses, and $\lambda \sim 0.22$ is the Wolfenstein parameter, given by the ratio $\langle\Phi\rangle / \Lambda_{c}=\langle\bar{\Phi}\rangle / \Lambda_{c}$. It is important for SRHND that at least some of the combinations $l_{i}+n_{p}$ and $n_{p}+n_{q}+\sigma$ take negative values; otherwise, the modulus signs could be dropped and the right-handed neutrino charges $n_{p}$ would cancel in $m_{\nu}$, as constructed using the seesaw formula (1.4). Therefore the choice of right-handed neutrino charges plays an important role in determining $m_{\nu}$ and each particular choice must be analysed separately.

We have studied several of the $U(1)_{H}$ charge assignments found in 23, which provide a natural account of the atmospheric and solar neutrinos via the LMA MSW effect. We have focused on choices that lead to all right-handed neutrino masses $m_{\nu_{R_{i}}}>10^{12} \mathrm{GeV}$, so they are not present in the thermal bath when the sphaleron processes enter into thermal equilibrium and GUT baryogenesis is in principle feasible ${ }^{2}$. For example, for diagonal textures we found that in the following cases GUT baryogenesis is viable

\begin{tabular}{|rrr|rrr|r|}
\hline$l_{1}$ & $l_{2}$ & $l_{3}$ & $n_{1}$ & $n_{2}$ & $n_{3}$ & $\sigma$ \\
\hline \hline-1 & 1 & 1 & 0 & $\frac{1}{2}$ & $-\frac{1}{2}$ & -1 \\
\hline-1 & 1 & 1 & $\frac{1}{2}$ & 0 & $-\frac{1}{2}$ & -1 \\
\hline$-\frac{1}{2}$ & $\frac{1}{2}$ & $\frac{1}{2}$ & $\frac{1}{2}$ & 0 & -1 & -1 \\
\hline
\end{tabular}

and as working examples of the democratic and the off-diagonal textures we have ${ }^{3}$

\begin{tabular}{|ccc|ccc|c|}
\hline$l_{1}$ & $l_{2}$ & $l_{3}$ & $n_{1}$ & $n_{2}$ & $n_{3}$ & $\sigma$ \\
\hline \hline-1 & 0 & 0 & $\frac{1}{2}$ & $\frac{1}{2}$ & -1 & -1 \\
\hline-1 & 0 & 0 & $\frac{1}{2}$ & $\frac{1}{2}$ & $-\frac{1}{2}$ & -1 \\
\hline-1 & 0 & 0 & $\frac{1}{2}$ & $\frac{1}{2}$ & 0 & -1 \\
\hline
\end{tabular}

\begin{tabular}{|rrr|ccc|c|}
\hline$l_{1}$ & $l_{2}$ & $l_{3}$ & $n_{1}$ & $n_{2}$ & $n_{3}$ & $\sigma$ \\
\hline \hline-2 & 0 & 0 & -2 & 1 & -1 & 1 \\
\hline-2 & 0 & 0 & -2 & -2 & 0 & 0 \\
\hline-2 & 0 & 0 & -1 & 1 & 0 & 0 \\
\hline
\end{tabular}

\footnotetext{
${ }^{2}$ The possibility of lighter right-handed neutrinos but with $\Delta L=1$ decays out of equilibrium at $T \lesssim$ $10^{12} \mathrm{GeV}$ will be considered in [11].

${ }^{3}$ Note that the third example of off-diagonal textures can be readily embedded in a grand unified $S U(5)$ theory, like the second one we considered in section 2.1.
} 


\subsection{Inverted hierarchy models}

This kind of models have been studied in [24] and are based on a mechanism similar to SRHND, but with dominance of two heavy right-handed neutrinos. A reversal of the SRHND conditions leads to an inverted hierarchical spectrum of the left-handed neutrinos, $m_{\nu_{3}} \ll m_{\nu_{2}} \sim m_{\nu_{1}}$, and bi-maximal mixing. As in the case of SRHND, the mechanism can naturally take place in models with a $U(1)_{H}$ family symmetry broken by two vector-like singlets with charges \pm 1 . We shall only be concerned about the leptonic sector, although these models could be extended to describe the quark masses as well, in a GUT scenario.

The only texture of the heavy Majorana neutrino matrix, $m_{R}$, that can lead to an inverted mass hierarchy is the off-diagonal one in eq. (2.21) [24]. Using for the Dirac neutrino mass matrix $m_{D}$ the same notation as in section 2.4 (see eq. (2.22) ), the seesaw formula (1.4) implies

$$
m_{\nu}=\left(\begin{array}{ccc}
\frac{d^{2}}{Y}+\frac{2 a a^{\prime}}{X} & \frac{d e}{Y}+\frac{a^{\prime} b+a b^{\prime}}{X} & \frac{d f}{Y}+\frac{a^{\prime} c+a c^{\prime}}{X} \\
\frac{d e}{Y}+\frac{a^{\prime} b+a b^{\prime}}{X} & \frac{e^{2}}{Y}+\frac{2 b b^{\prime}}{X} & \frac{e f}{Y}+\frac{b^{\prime} c+c b^{\prime}}{X} \\
\frac{d f}{Y}+\frac{a^{\prime} c+a c^{\prime}}{X} & \frac{e f}{Y}+\frac{b^{\prime} c+c b^{\prime}}{X} & \frac{f^{2}}{Y}+\frac{2 c c^{\prime}}{X}
\end{array}\right) v^{2}
$$

In order to achieve an inverted hierarchy one should require that the $\mathcal{O}\left(\frac{1}{X}\right)$ terms dominate over the $\mathcal{O}\left(\frac{1}{Y}\right)$ ones (which is the opposite of the SRHND condition), and that either $a^{\prime}, b, c \gg a, b^{\prime}, c$ or $a^{\prime}, b, c \ll a, b^{\prime}, c^{\prime}$. In the first case one finds the following $\nu_{L}$ masses

$$
m_{\nu_{3}} \approx \frac{(b-c)^{2}}{b^{2}+c^{2}} \frac{1}{Y} v^{2} \quad m_{\nu_{1}} \approx m_{\nu_{2}} \approx \frac{a^{\prime} \sqrt{b^{2}+c^{2}}}{X} v^{2} .
$$

Introducing a $U(1)_{H}$ flavour symmetry, the conditions for achieving the required offdiagonal texture of $m_{R}$ and an inverted hierarchical spectrum of the left-handed neutrinos translate into conditions of the $U(1)_{H}$ charges for the different fields. Many working examples can be found in [24], of which we have analysed some. In particular, we have obtained that the three right-handed neutrinos are heavier than $10^{12} \mathrm{GeV}$ and therefore GUT baryogensis is viable for the following choices of the $U(1)_{H}$ charges

\begin{tabular}{|ccc|ccc|c|}
\hline$l_{1}$ & $l_{2}$ & $l_{3}$ & $n_{1}$ & $n_{2}$ & $n_{3}$ & $\sigma$ \\
\hline \hline-2 & 3 & 3 & 2 & -2 & 0 & 0 \\
\hline-3 & 1 & 1 & 3 & -2 & 1 & -2 \\
\hline-3 & 3 & 3 & 3 & -3 & -1 & 1 \\
\hline
\end{tabular}

where the charges $l_{i}, n_{i}, \sigma$ correspond to the $l_{L}, \nu_{R}, S$ fields, as in the case of SRHND.

\section{Conclusions}

We have found that the GUT-baryogenesis mechanism proposed in [ $U(1)_{H}$ grand unified models, with appropriate choices of the $U(1)_{H}$ charges such that the right-handed neutrinos are not very hierarchical, and all of them heavier than $10^{12} \mathrm{GeV}$.

In models with grand unified gauge group $S O(10)$ and Pati-Salam, the mechanism does not work, since $\Delta L=1$ right-handed neutrino decays are in equilibrium below $10^{12} \mathrm{GeV}$, 
together with the $B+L$ violating sphaleron interactions. This is because in those models the Dirac neutrino mass matrix is somehow related to the up quark mass matrix, and thus it is very hierarchical. Since the observed hierarchy in the light neutrino masses is not so strong, $\sqrt{\Delta m_{\text {atm }}^{2}} / \sqrt{\Delta m_{\text {sol }}^{2}} \sim 0.1$, this implies a hierarchy on the right-handed neutrinos, so one ore more of them result with a small mass, lighter than $10^{12} \mathrm{GeV}$, and their lepton number violating decays are in thermal equilibrium. Although we have only analysed some particular models, this feature seems generic for such gauge groups, independently of the flavour symmetry, as far as neutrino masses are generated via the minimal seesaw mechanism, i.e., neglecting $S U(2)$ triplet vevs.

From a more phenomenological perspective, we have also found that GUT baryogensis works for many choices of the $U(1)_{H}$ charges in models with single right-handed neutrino dominance, and in models where two right-handed neutrinos dominate an lead to an inverted hierarchy of the left-handed neutrinos. Again, these models allow all heavy right-handed neutrinos with masses larger than $10^{12} \mathrm{GeV}$.

\section{Acknowledgements}

We are specially grateful to Sacha Davidson for many interesting comments and a careful reading of the manuscript. We also thank Martin Hirsch for discussions, and all the organisers for a very interesting workshop. This work was partially supported by the Spanish MCyT grants BFM2002-00345 and FPA2001-3031, by the TMR network contract HPRN-CT-2000-00148 of the European Union and by the Generalitat Valenciana grant CTIDIB/2002/24.

\section{References}

[1] A.D. Sakharov, Pis'ma Zh. Eksp. Teor. Fiz. 5, 32 (1967) English translate: [A.D. Sakharov, JETP Lett. 5, 24 (1967)]; S. Weinberg, Phys. Rev. Lett. 42, 850 (1979); A.Y. Ignatev, N.V. Krasnikov, V.A. Kuzmin and A.N. Tavkhelidze, Phys. Lett. B 76, 436 (1978); M.

Yoshimura, Phys. Rev. Lett. 41, 281 (1978) [Erratum: 43, 746 (1979)]

[2] V.A. Kuzmin, V.A. Rubakov, M.E. Shaposhnikov, Phys. Lett. B 155, 36 (1985); N.S. Manton, Phys. Rev. D 28, 2019 (1983); F.R. Klinkhammer, N.S. Manton Phys. Rev. D 30, 2212 (1984); E.W. Kolb, M.S. Turner, The early Universe (Addison-Wesley, 1990)

[3] P. Arnold, L. McLerran, Phys. Rev. D 36, 581 (87); D 37, 1020 (1988); A. Bochkarev, M.E. Shaposhnikov, Mod. Phys. Lett. , A 2, 417 (1987); A 2, 921 (1987); S.Yu. Khlebnikov, M.E. Shaposhnikov, Nucl. Phys. B 308, 885 (1988); D. Bödeker, G.D. Moore, K. Rummukainen, Phys. Rev. D 61, 056003 (2000)

[4] M. Fukugita, T. Yanagida, Phys. Rev. Lett. 89, 131602 (2002) [arXiv:hep-ph/0203194]

[5] Super-Kamiokande Collaboration (Y. Fukuda et al.), Phys. Rev. Lett. 81, 1562 (1998) [arXiv:hep-ex/9807003]

[6] K2K Collaboration, (S.H. Ahn et al.) Phys. Rev. Lett. 90, 041801 (2003) [arXiv:hep-ex/0212007]

[7] Super-Kamiokande Collaboration (S. Fukuda et al.), Phys. Lett. B 539, 179 (2002) [arXiv:hep-ex/0205075] 
[8] SNO Collaboration (Q.R. Ahmad et al.) Phys. Rev. Lett. 89, 011302 (2002) [arXiv:nucl-ex/0204009]

[9] KamLAND Collaboration (K. Eguchi et al.) Phys. Rev. Lett. 90, 021802 (2003) [arXiv:hep-ex/0212021]

[10] M. Gell-Mann, P. Ramond, and R. Slansky, Supergravity (North Holland,1979)]; T. Yanagida, Prog. Theor. Phys., 64, 1103 (1980)]; R.N. Mohapatra and G. Senjanović, Phys. Rev. Lett. 44, 912 (1980)

[11] S. Davidson, J.A. Lopez-Perez, N. Rius, in preparation.

[12] CHOOZ Collaboration (M. Apollonio et al.), Phys. Lett. B 466, 415 (1999) [arXiv:hep-ex/9907037]

[13] H. Fritzsh, Phys. Lett. B 70, 436 (1977); C.D. Froggatt, H.B. Nilsen, Nucl. Phys. B147, 277 (1979); J. Harvey, P. Ramond, D. Reiss, Phys. Lett. B 92, 309 (1980); C. Wetterich, Nucl. Phys. B 261, 461 (1985); P. Kaus, S. Meshkov, Mod. Phys. Lett. A 3, 1251 (1988); L. Ibáñez, G.G. Ross, Phys. Lett. B 332, 100 (1994); P. Binetruy, P. Ramond, Phys. Lett. B 350, 49 (1995) [arXiv:hep-ph/9412385]

[14] D.V. Nanopoulos, S. Weinberg, Phys. Rev. D 20, 2484 (1979); S.M. Barr, G. Segre, H.A. Weldon, Phys. Rev. D 20, 2494 (1979)

[15] W. Buchmüller, T. Yanagida, Phys. Lett. 445, 339-402 (1999); W. Buchmüller, M. Plümacher, Int. J. Mod. Phys., A 15, 5047-5086 (2000) [arXiv:hep-ph/0007176], and references therein.

[16] J. Sato, T. Yanagida, Nucl. Phys. Proc. Suppl. 77, 293 (1999) [arXiv:hep-ph/9809307]

[17] G. Altarelli, F. Feruglio, in Neutrino Mass, Springer Tracts in Modern Physics, and references therein.

[18] B. Bajc, G. Senjanovic and F. Vissani, [arXiv:hep-ph/0210207] y Hirsch ,Valle ...

[19] S.M. Barr, S. Raby, Phys. Rev. Lett. 79, 4748 (1997); C.H. Albright, S.M. Barr, Phys. Rev. D 58, 013002 (1998); C.H. Albright, K.S. Babu, S.M. Barr, Phys. Rev. Lett. 81, 1167 (1998); C.H. Albright, S.M. Barr, Phys. Rev. Lett. 85, 244 (2000); Phys. Rev. D 62, 093008 (2000); Phys. Rev. D 64, 073010 (2001); C.H. Albright, talk presented at Neutrinos and Implications for Physics Beyond the Standard Model, Stony Brook, New York, 11-13 Oct 2002 [arXiv:hep-ph/0212090]

[20] G.C. Branco, R. Gonzalez Felipe, F.R. Joaquim, M.N. Rebelo, Nucl. Phys. B 640, 202 (2002) [arXiv:hep-ph/0202030]

[21] J.C. Pati, A. Salam, Phys. Rev. D 10, 275 (1974)

[22] S.F. King, M. Oliveira, Phys. Rev. D 63, 095004 (2001) [arXiv:hep-ph/0009287]; T. Blazek, S.F. King, J.K. Parry, JHEP 0305016 (2003) [arXiv:hep-ph/0303192]

[23] S.F. King, Nucl. Phys. B 576, 85-105 (2000) [arXiv:hep-ph/9912492], and references therein.

[24] S.F. King, N. Nimai Singh, Nucl. Phys. B 596, 81 (2001) [arXiv:hep-ph/0007243] 